\title{
Abstracts of the 15th Annual ENETS Conference for the Diagnosis and Treatment of Neuroendocrine Tumor Disease
}

March 7-9, 2018, Barcelona, Spain

Guest Editor

Nicholas Reed, Glasgow 


\section{Disclosure Statement Guest Editor}

Prof. Nicholas Reed has attended advisory boards for Novartis, AAA, Ipsen and Eisai. He has also received travel bursaries from Ipsen, and Novartis.

S. Karger

Medical and Scientific Publishers

Basel $\cdot$ Freiburg $\cdot$ Paris $\cdot$ London .

New York $\cdot$ Chennai $\cdot$ New Delhi

Bangkok $\cdot$ Beijing $\cdot$ Shanghai $\cdot$ Tokyo .

Kuala Lumpur $\cdot$ Singapore $\cdot$ Sydney
Disclaimer

The statements, opinions and data contained in this publication are solely those of the individual authors and contributors and not of the publisher and the editor(s). The appearance of advertisements in the journal is not a warranty, endorsement, or approval of the products or services advertised or of their effectiveness, quality or safety. The publisher and the editor(s) disclaim responsibility for any injury to persons or property resulting from any ideas, methods, instructions or products referred to in the content or advertisements.

Drug Dosage

The authors and the publisher have exerted every effort to ensure that drug selection and dosage set forth in this text are in accord with current recommendations and practice at the time of publication. However, in view of ongoing research, changes in government regulations, and the constant flow of information relating to drug therapy and drug reactions, the reader is urged to check the package insert for each drug for any change in indications and dosage and for added warnings and precautions. This is particularly important when the recommended agent is a new and/or infrequently employed drug.
All rights reserved.

No part of this publication may be translated into other languages, reproduced or utilized in any form or by any means, electronic or mechanical, including photocopying, recording microcopying, or by any information storage and retrieval system, without permission in writing from the publisher or, in the case of photocopying, direct payment of a specified fee to the Copyright Clearance Center (see 'General Information').

(c) Copyright 2018 by S. Karger AG,

P.O. Box, CH-4009 Basel (Switzerland) e-ISBN 978-3-318-06354-7

\section{KARGER}




\section{Contents}

\section{A Basic Science - Signaling Pathways, Receptors, Biomarkers}

A01 Study of Cell Cycle Protein Expression Pattern in Bronchial Carcinoids: A New Potential Target for Medical Therapy? Bresciani $G$ et al.

A02 Analysis of Gene Expression Patterns of Rectum Neuroendocrine Tumors (NET) Versus Healthy Tissue Reveals Alterations in the MAPK and WNT Signaling Pathways Briest $\mathrm{F}$ et al.

A03 Expression of FOXM1 in G3 Neuroendocrine Tumors (NET G3) and G3 Neuroendocrine Carcinomas (NEC G3) of the Pancreas and the Intestine

Briest $\mathrm{F}$ et al.

A04 Analysis of MET and RET Expression in Patients with Pancreatic Neuroendocrine Tumors Receiving Everolimus Cella CA et al.

A05 Are elFs Ingredients for Neuroendocrine Tumorigenesis? Gantenbein $\mathrm{N}$ et al.

A06 Identification and Validation of the Angiotensin II Receptor Type 1 as a Possible Anti-Cancer Target in Neuroendocrine Tumors Grötzinger $\mathrm{C}$ et al.

A07 Somatostatin Analogs and mTOR Inhibitors as Radioprotectors or Radiosensitizers in Neuroendocrine Tumor Cells Grötzinger C et al.

A08 Expression of Somatostatin Receptors in Neuroendocrine Tumors of the Lungs with Various Degrees of Malignancy Gurevich L et al.

A09 Ghrelin O-Acyltransferase (GOAT) Enzyme and Ghrelin Receptor GHSR1 a as Putative Prognosis Markers and Therapeutic Targets in Gastroenteropancreatic Neuroendocrine Tumors Herrera-Martínez AD et al.

A10 Long Non-Coding RNA H19 Promotes Malignant Proliferation and Metastasis in Pancreatic Neuroendocrine Neoplasms Ji $\mathrm{M}$ et al.

A11 Stromal Immune Contexture Predicts Disease-Free Survival in Gastro-Entero-Pancreatic Neuroendocrine Neoplasms (GEP-NENs) Milione $\mathrm{M}$ et al.
A12 Decreased Serum Amyloid A1 as Potential Diagnostic Marker for Gastro-Entero-Pancreatic Neuroendocrine Neoplasms $\mathrm{Na} \mathrm{H}$ et al.

A13 IMMUNeOCT Study: The Role of Octreotide LAR in Modulating the Immune Response in Patient with Neuroendocrine Tumors. EudraCT Number 2017-001613-83

Napolitano $\mathrm{M}$ et al.

A14 Potential Role of SST5TMD4-Derived Peptides in the Malignancy of Neuroendocrine Tumors and Other Endocrine-Related Cancer Cells

Pedraza-Arévalo $S$ et al.

A15 177Lu-TOC Inhibits Cell Proliferation by Inducing Autophagy and G2/M Cell Arrest in Neuroendocrine Prostate Cancer Cell Qiu F et al.

A16 Differential Signalling Pathways Drive Therapeutic Resistance in Neuroendocrine Tumors

Romano D et al.

A17 Tumor Necrosis Factor-Related Apoptosis-Inducing Ligand (TRAIL) Receptor Expression in Patients with Neuroendocrine Neoplasia

Sättele R et al.

A18 Can Insulin-Like Growth Factor 1 (IGF-1), IGF-1 Receptor, Connective Tissue Growth Factor and Ki-67 Labelling Index Have a Prognostic Role in Pulmonary Carcinoids?

Tsolakis A et al.

A19 Systematic Evaluation of the Immune Microenvironment of Neuroendocrine Tumors (NET)

Vesely C et al.

A20 The Anticancer Mechanism of Metformin in Pancreatic Neuroendocrine Tumor Cells

Vitali E et al.

A21 Expression and Clinical Significance of Vascular Endothelial Growth Factor D in Gastroenteropancreatic Neuroendocrine Neoplasms

Zhang Y et al.

A22 Expression and Clinical Significance of Vascular Endothelial Growth Factor Receptor 3 (VEGFR3) and Phospho-VEGFR3 in Gastroenteropancreatic Neuroendocrine Neoplasms Zhang Y et al.
KARGER

(C) 2018 S. Karger AG, Basel 


\section{B Basic Science - Genetics, Epigenetics, miRNAs, OMICS}

B01 Abstract Withdrawal

B02 Genome-Wide DNA Methylation Profiling of Pancreatic Neuroendocrine Tumors

Boons $\mathrm{G}$ et al.

B03 BRAF-V600E Driven Mutations in Grade 3 Neuroendocrine Carcinomas of Colon Origin: Results from Genomic/Epigenomic Profilings and Patient-Derived Mouse Model Capdevila J et al.

B04 Genomic and Transcriptomic Characterization of Aggressive Well Differentiated Pancreatic Neuroendocrine Tumors (WD PanNET) Cros J et al.

B05 Mitochondrial or Aerobic Glycolysis Oriented Metabolism May Define Pancreatic Neuroendocrine Tumors (PanNET) Resistance to mTOR-Targeting Therapies

Cros J et al.

B06 Epigenetic Changes in DAXX and/or ATRX Negative Pancreatic Neuro-Endocrine Tumors

Di Domenico A et al.

B07 Finding the Ways to Determine the Prognosis of Pulmonary Carcinoids with Certain Genes/Markers in Pakistan Fatima A et al.

B08 Retrospective Analysis of Gastroenteropancreatic Neuroendocrine Neoplasms and the Role of GSK-3 $\beta$ Expression in Neuroendocrine Neoplasms $\mathrm{Hu} \mathrm{Y}$ et al.

B09 Analysis of the Proteomics of Serum Circulating Exosomes in Gastro-Entero-Pancreatic Neuroendocrine Neoplasms Jian-An B et al.

B10 A Novel Classification of Pancreatic Neuroendocrine Tumors (pNETs) Guided by Genomics

Lawrence B et al.

B11 Endocan Expression Is Correlated with Poor Progression-Free Survival in Patients with Pancreatic Neuroendocrine Tumors Lin LY et al.

B12 Chemoprevention with Enalapril and Aspirin in Men 1(+/T) Knockout Mouse Model Manoharan J et al.

B13 Genetic Analysis Identifies Subgroups of Small WellDifferentiated Pancreatic Neuroendocrine Tumors with Different Risk for Liver Metastases

Pea A et al.

B14 Enhancing Diagnostic Precision Through Combined Genomic and Histopathological Analysis of Pancreatic Neuroendocrine Tumors

Robb T et al.

B15 Merkel Cell Carcinomas in New Zealand: Virus or Ultra Violet? Robb T et al.

B16 High Rate of Copy-Number Alterations in Gastrointestinal and Pancreatic Neuroendocrine Tumors with Unidentified Driver Mutations

Tirosh A et al.
B17 Ultra-Deep Targeted Resequencing Reveals Recurrent DAXX and CYFIP2 Mutations and Implicates Novel Pathways in Pancreatic Neuroendocrine Tumors

Vandamme $\mathrm{T}$ et al.

B18 Screening of Differentially Expressed Genes in Gastric Neuroendocrine Neoplasms Based on Human Transcriptome Array Wei Y et al.

B19 Molecular Characterization of Primary and Metastatic Pancreatic Neuroendocrine Tumors

Yang $\mathrm{KC}$ et al.

\section{Basic Science - In Vitro Models, Tumor Growth, CTCs}

C01 The Effect of Temozolomide on Pancreatic Neuroendocrine Tumors in Vitro and Role of MGMT and MMR System in Temozolomide Resistance

Blazevic A et al.

C02 The BON-SSTR2 Chicken Chorioallantoic Membrane (CAM) Model for the Analysis of Lu-17-DOTATOC Sensitizing Agents Briest $\mathrm{F}$ et al.

C03 Single Cell Copy Number Variation Analysis (CNV) of Circulating Tumor Cells (CTCs) in Neuroendocrine Tumor (NET) Patients Childs A et al.

C04 Using Adult Stem-Cell Derived Organoids to Model Neuroendocrine Tumor Growth

Dayton $\mathrm{T}$ et al.

C05 Effects of Multi-Receptor Targeting Drugs in Neuroendocrine Tumors Using 3D Cell Culture

Herrera-Martínez AD et al.

C06 Potential Effects of Ketoconazole on ACTH-Producing and Non-ACTH-Producing Neuroendocrine Tumors

Herrera-Martínez AD et al.

C07 The Neuroendocrine Phenotype, Genomic Profile, and Therapeutic Sensitivity of GEPNET Cell Lines Hofving $\mathrm{T}$ et al.

C08 Preclinical Testing of SSA Compounds in a Model of Pancreatic Neuroendocrine Tumors to Optimize Scheduling and Drug Exposition Martínez-López A et al.

C09 Autophagy Process in Pancreatic Neuroendocrine Tumor Cells Matrood S et al.

C10 CXCR4 Expression on Circulating Tumor Cells (CTCs) and Their Association with Bone Metastases in Patients with Neuroendocrine Tumors (NET)

Rizzo FM et al.

C11 Epigenetic Modulation of Autophagy in Pancreatic Spheroid Cells

Matrood S et al.

C12 Development of Pre-Clinical NEN Models - Limitations and Opportunities

Schrader J et al. 
C13 Histone Replacement in Cancer: Dissecting the Role of H3.3 Chaperones in Pancreatic Tumorigenesis

Sposito T et al.

C14 Preliminary Evaluation of Influence of Different Targeted Drugs of Vascular Endothelial Growth Factor Signaling Pathway on the Proliferation of Neuroendocrine Neoplasm Cell Lines

Zhang $\mathrm{Y}$ et al.

\section{Epidemiology/Natural History/Prognosis- Registries, Nationwide and Regional Surveys}

D01 Treatment Outcomes of Patients with Mixed Neuroendocrine Non-Neuroendocrine Neoplasms (MiNEN)

Apostolidis L et al.

D02 Neuroendocrine Tumors: An Australian Experience Ashoka Menon V et al.

D03 Recognition of Primary Neuroendocrine Tumors - A Challenge Athar A et al.

D04 First Patient Survey about Tumor - Associated Fatigue in NEN Banz S et al.

D05 Direct Cost of Metastatic Gastroenteropancreatic Neuroendocrine Tumors (GEP-NET) Grade 1 or 2 (G1/G2) in Relation to Time Since Diagnosis Shows Growing Importance of Somatostatin Analogues (SSA)

Berthon A et al.

D06 Long-Acting Somatostatin Analogue (LA-SSA) Treatment Durations in Patients Diagnosed with Metastatic Gastroenteropancreatic Neuroendocrine Tumors (GEP-NET) Grade 1 or 2 (G1/G2) in Sweden

Berthon A et al.

D07 The ENETS Registry: First Results of a Collaborative Effort Including over 12.000 Patients with Neuroendocrine Neoplasms (NENs) from 7 European Countries

Borbath I et al.

D08 Pattern and Risk Factors for Distant Metastases in Gastrointestinal Neuroendocrine Neoplasms: A PopulationBased Study

Cai W et al.

D09 Neuroendocrine Tumors in the Chilean Population Carrillo D et al.

D10 Thrombotic Risk in Gastroenteropancreatic Neuroendocrine Tumors: A Single Centre Experience

Cavalcoli FA et al.

D11 Endocrine Paraneoplastic Syndromes in Patients with Neuroendocrine Neoplasms

Chatzellis E et al.

D12 Diagnostic and Clinical Management of Pancreatic Neuroendocrine in MEN1 Syndrome Chiloiro $S$ et al

D13 Screening Benefits in MEN1-Associated Pituitary Adenomas Chiloiro $\mathrm{S}$ et al.

D14 Current Educational Strategies Used by Nurses Caring for NET Patients: Electronic Survey across 25 Countries

Davies $\mathrm{P}$ et al.
D15 Trends in Diagnosis of Gastroenteropancreatic Neuroendocrine Tumors (GEP-NETs) in India: A Report of Multicentre Data from a Web Based Registry

Desai G et al.

D16 Single-Institutional Review of Non-Pancreatic GI Neuroendocrine Tumors

El Chami A et al.

D17 Retrospective Analysis of 228 Digestive System Neuroendocrine Tumor Patients from the Northwest of China

Fan Y et al.

D18 The Trend of Using CT to Diagnose the Gastroenteropancreatic Neuroendocrine Neoplasms: Results of a Nation-Wide Retrospective Epidemiology Study in China Fan J et al.

D19 Finding the Options of Good Prognosis for Ampullary Neuroendocrine Tumors

Fatima A et al.

D20 Carcinoid Syndrome Open Questions - Evaluations from a Real Life Setting

Fijalkowski R et al.

D21 Carboplatin-Etoposide Chemotherapy (CB-ET) for Patients Diagnosed with Advanced Extra-Pulmonary (EP) Poorly Differentiated (PD) Neuroendocrine Carcinoma (NEC): Findings from a European Neuroendocrine Tumor Society Centre of Excellence

Frizziero $\mathrm{M}$ et al.

D22 Data on Healthcare Resource Utilization (HCRU) and Treatment Modalities in Patients (Pts) with Advanced (adv), Well-Differentiated (WD) Neuroendocrine Tumors (NETs) of Gastrointestinal (GI) or Lung Origin in Finland Gaasvik L et al.

D23 Reassessment of Risk Factors Associated with Locoregional Lymph Nodal Metastases in Well-Differentiated Appendiceal Neuroendocrine Neoplasms

Galanopoulos $\mathrm{M}$ et al.

D24 Is the Classification of Functioning-PanNET/Non-Functioning PanNET Properly? Insulinoma/Non-Insulinoma Is More Suitable to Distinguish the Clinicopathological and Prognosis Difference of Localized Insulinoma

Gao H et al.

D25 The Latest Exploration of Staging and Prognostic Classification for Pancreatic Neuroendocrine Tumors Gao S et al.

D26 Ki-67 to Predict Recurrence and Survival of Pancreatic Neuroendocrine Tumors

Genc C et al.

D27 1-Year Survival Rates for Neuroendocrine Tumor Patients in England

Genus T et al.

D28 Incidence and Prevalence of Neuroendocrine Tumors in England Genus T et al.

D29 Metachronous Primary Cancers in Neuroendocrine Tumor Patients

Genus T et al. 
D30 Incidental Histological Diagnosis of Small Pancreatic Neuroendocrine Tumors: Are We Underestimating Their Incidence?

Giannone F et al.

D31 Vitamin D, Bone Mineral Density and Fracture Risk Assessment in Patients with Neuroendocrine Tumors

Grimaldi F et al.

D32 Diffuse Idiopathic Pulmonary Neuroendocrine Cell Hyperplasia (DIPNECH): Characterisation of Prevalence and Prognosis in the Pulmonary Carcinoid Population

Hayes A et al.

D33 Predictive Markers of Gastroenteropancreatic Neuroendocrine Tumors (GEP-NETS)

Issaeva $S$ et al.

D34 Neuroendocrine Tumors Committee of Reference Hospital: Decision-Making Process on the Diagnostic and Therapeutic Approach to Patients with Neuroendocrine Tumors Jimenez-Fonseca $\mathrm{P}$ et al.

D35 The Results of Screening of the Syndrome of Cushing in the Republic of Uzbekistan Khalimova $\mathrm{Z}$ et al.

D36 Retrospective Epidemiologic Study of Medullary Cancer of Thyroid Gland in Hospital - Based Registry in Moscow Cancer Centre

Komarova L et al.

D37 Clinical Significance of Incidental Detection of Appendicular Neuroendocrine Tumors in Sri Lanka

Lal DPCK et al.

D38 Subtype Classification and Clinicopathological Features of Gastric Neuroendocrine Neoplasms: 400 Cases of Multicenter Retrospective Analysis

Li YL et al.

D39 Nutritional Assessment and Vitamin Deficiencies in Patients with NETs

Lim S et al.

D40 A Retrospective Analysis of 344 Cases with Neuroendocrine Neoplasms

Liu Z et al.

D41 Assessment of Quality of Life in Chinese Patients with Gastroenteropancreatic Neuroendocrine Tumors Liu Q et al.

D42 Risk Factors Affecting Prognosis in Metachronous Liver Metastases from WHO Classification G1 and G2

Gastroenteropancreatic Neuroendocrine Tumors after Initial R0 Surgical Resection

Lyu Y et al.

D43 Analysis of Clinical Pathology Features and Prognostic Factors of 20 Patients with Appendiceal Neuroendocrine Neoplasms Mao W et al.

D44 Development of a Predictive Prognostic Nomogram for the Neuroendocrine Carcinomas of Rectum Based on a Large Population-Based Exploration Mao W et al.
D45 Patterns of Recurrence after Resection for Pancreatic Neuroendocrine Tumors: Who, When, and How? Masini G et al.

D46 Can Neutrophil-to-Lymphocyte Ratio (NLR) Predict Clinical Outcomes of Pancreatic Neuroendocrine Tumors (PanNETs)? Massironi S et al.

D47 Eterogeneity of Duodenal Neuroendocrine Tumors: A MultiCentre Experience In Italy Massironi S et al.

D48 The Impact of NETwerk on the Multidisciplinary Tumor Boards Maurissen I et al.

D49 Are Current ENETS Guidelines for Surgery of Small $(<2 \mathrm{~cm})$ Pancreatic Neuroendocrine Neoplasms Implemented in German Surgical Community?

Mintziras I et al.

D50 Perioperative Outcomes of Surgery for Pancreatic Neuroendocrine Neoplasms in Germany Mintziras I et al.

D51 Survival and Prognosis in Patients with Duodeno-Pancreatic Neuroendocrine Tumors in Multiple Endocrine Neoplasia Type 1 Syndrome

Modica $\mathrm{R}$ et al.

D52 The Condition of Carbohydrate Exchange in New Revealed Patients with Cushing's Syndrome Narimova G et al.

D53 Prevalence of Carcinoid Syndrome (CS) in the European Union (EU) Nayroles G et al.

D54 Approaching NETs from Many Angles: A Medical Research Paradigm to Challenge New Zealand's Research Traditions Parker K et al.

D55 The NETwork! Registry Suggests Increasing Incidence in a National Study of Neuroendocrine Cancer in New Zealand (NZ) Parker K et al.

D56 Clinicopathological Features of 63 Cases with Type 3 Gastric Neuroendocrine Tumors Qiu XD et al.

D57 Remote Tracking of Symptoms, QoL and Wellbeing within the New Wales NET Service Reynolds $\mathrm{M}$ et al.

D58 High Hepatic Tumor Burden and History of Cardiovascular Comorbidities Are Associated with Carcinoid Heart Disease: A Multicenter and Multinational Study Riechelmann $\mathrm{R}$ et al.

D59 Patient Survey Devoted to Characterizing Experience and Expectations of Patients with Neuroendocrine Tumors (NET) Sarabi $\mathrm{M}$ et al.

D60 Gastroenteropancreatic Neuroendocrine Neoplasms (GEP-NENs) in the Czech NEN Registry 2009-2016: Histologic Spectrum and Clinicopathological Features Sedláčková E et al. 
D61 The Risk of Secondary Primary Colorectal Adenocarcinomas Is Not Increased among Patients with Gastroenteropancreatic Neuroendocrine Tumors - A Nationwide Population Based Study Stemann Lau T et al.

D62 Diagnosing and Managing MEN1-Related pNETs in Israel: A Specialist Center Experience Uri I et al.

D63 Frequency, the Characteristic of Diseases of Thyroid Gland at Children and Teenagers According to Negotiability during 5 Years

Urmanova $\mathrm{Y}$ et al.

D64 The Frequency of Patients with Giant Pituitary Tumors from Retrospective Data

Urmanova Y et al.

D65 Is the EORTC QLQ-QNET21 Optimal for Patients with Neuroendocrine Tumors?

Van Leeuwaarde R et al.

D66 Excellent Survival Outcomes for NET Patients Treated within NETwerk, a Collaborative ENETS Center of Excellence Vandamme $\mathrm{T}$ et al.

D67 Prognostic Factors of Pulmonary Carcinoid Tumors Vesterinen $\mathrm{T}$ et al.

D68 Self-Reported Side Effects in Neuroendocrine Tumor (NET) Patients Prescribed Somatostatin Analogues - The Role for Specialist Dietitians and Nurses Whyand $\mathrm{T}$ et al.

D69 Financial Toxicity in Patients with Neuroendocrine Tumor Disease. A so far Underestimated "Side Effect" of Cancer in Germany?

Winkler EC et al.

D70 How the Rare See Their Care: Perceptions of Care and Patient Reported Outcomes in People Living with Neuroendocrine Tumors

Wyld $\mathrm{D}$ et al.

D71 Clinicopathologic Characteristics of Chinese Patients with Gastroenteropancreatic Neuroendocrine Neoplasms: Results of a Nation-Wide Retrospective Epidemiology Study Yu $\mathrm{P}$ et al.

D72 Evaluation of Multidisciplinary Tumor Board Decisions in the Management of Neuroendocrine Tumors: Impact of Expert Centers

Zandee $\mathrm{W}$ et al.

D73 A Single-Center Retrospective Analysis of Clinicopathologica Characteristics of Gastroenteropancreatic Large Cell Neuroendocrine Neoplasms (GEP-LCNEC)

Zhang $\mathrm{P}$ et al.

D74 Clinical Study on High-Risk Factors Leading to Recurrence of Well-Differentiated Pancreatic Neuroendocrine Neoplasms after Surgery

Zhang P et al.

D75 Clinicopathological Features and Prognosis of Colonic Neuroendocrine Neoplasms in Different Races

Zhang $\mathrm{Y}$ et al.
D76 The Exact Prognostic Significance of Lymphatic Metastasis of Jejunoileal Neuroendocrine Tumors

Zhang $\mathrm{Y}$ et al.

D77 Long-Acting Somatostatin Analogue (LA-SSA) Treatment Patterns in Patients Diagnosed with Metastatic Gastroenteropancreatic Neuroendocrine Tumors (GEP-NET) Grade 1 or $2(\mathrm{G} 1 / \mathrm{G} 2)$ in Sweden

Berthon $\mathrm{A}$ et al.

\section{E Pathology-Grading, Staging}

E01 The Number of Positive Nodes Accurately Predicts Recurrence after Pancreaticoduodenectomy for Nonfunctioning Neuroendocrine Neoplasms

Andreasi $\mathrm{V}$ et al.

E02 Immunohistochemical Profiles in 81 Patients with Resected Insulinomas

Andreassen $\mathrm{M}$ et al.

E03 Poorly Differentiated Neuroendocrine Carcinomas of the Colon: A Clinicopathological Study

Boeck I et al.

E04 Expanding the WHO 2017 Classification to Gastrointestinal Tumors: Real-World Data from the R-GETNE Registry

Carmona-Bayonas A et al.

E05 Clinicopathological Features of the High Grade Pancreatic Neuroendocrine Neoplasms

Delektorskaya $\mathrm{V}$ et al.

E06 Well-Differentiated Neuroendocrine Lesions in Inflammatory Bowel Disease

Dhall D et al.

E07 High Grade (G3) Gastro-Entero-Pancreatic Neuroendocrine Neoplasms: Should the New WHO Classification Apply to All? Furnace $\mathrm{M}$ et al.

E08 Novel Recurrence Risk Stratification of Resected Pancreatic Neuroendocrine Tumor

Gao $\mathrm{H}$ et al.

E09 Phosphohistone H3 (PHH3) Immunohistochemical Staining Outperforms Conventional H\&E Mitotic Count in Classifying Pulmonary Carcinoids

Luong TV et al.

E10 Histopathological Revision for Gastroenteropancreatic Neuroendocrine Neoplasms in Expert Centers: Does It Make the Difference?

Merola E et al.

E11 Ki-67 and Presence of Liver Metastases Predicts Progression in Pancreatic Neuroendocrine Neoplasms (pNENs)

Milione $\mathrm{M}$ et al.

E12 EUS-FNA in the Diagnosis of Pancreatic Neuroendocrine Tumors and Accuracy of Ki-67 Measurement on Cytological Samples: The Turin Experience

Rizza S et al.

E13 Intrinsic Contact between T Classification and N Classification in Resected Well-Moderate Differential Locoregional Pancreatic Neuroendocrine Neoplasms

$\mathrm{Xu}$ JM et al. 


\section{F Biomarkers}

F01 Tumor Necrosis of Primary Malignancy Affects Survival and Outcome in Patients with Liver Metastases from Neuroendocrine Tumors

Atanasov G et al.

F02 Significance of Biochemical Testing in Pancreatic Neuroendocrine Tumors

Athar A et al.

F03 When Is Right Hemicolectomy Really Indicated for Pediatric Appendiceal NETs?

Bajciova $\mathrm{V}$ et al.

F04 Systemic Markers of Inflammation in Neuroendocrine Tumors (NETs) and Outcomes with Everolimus: A Pooled Analysis from the Randomized, Phase 3 RADIANT-3 and RADIANT-4 Trials Chan DL et al.

F05 A Prospective Nordic Study on the Use of Chromogranin A for the Prediction of Progression in Patients with Pancreatic and Small Intestinal Neuroendocrine Tumors

Dam G et al.

F06 The Clinical Value of Circulating Transcript Analysis (NETest) during Follow-Up of Resected Well-Differentiated Pancreatic Neuroendocrine Tumors

Genc $\mathrm{C}$ et al.

F07 Prognostic Value of the Different Pre-Treatment Biomarkers for Patients with Neuroendocrine Tumors

Grigorescu RR et al.

F08 Plasma Protein Kallikrein-14 Strongly Predicts Pronounced Chromogranin A (CgA) Response in Small Intestinal Neuroendocrine Tumor (NET) Patients after Somatostatin Analog (SSA) Treatment: The Nordic EXPLAIN Biomarker Study Kjellman $\mathrm{M}$ et al.

F09 The Role of Subcutaneous Octreotide Suppression Test in Detecting Patients with Neuroendocrine Neoplasms: A Pilot Study

Kruljac I et al.

F10 Assessment of the Clinical Utility of the NETest in a US RegistryBased Study

Liu E et al.

F11 Tumor Microenvironment in Merkel Cell Carcinoma: Association with Merkel Cell Polyomavirus and Clinico-Pathological Features in a Retrospective Cohort Study

$\mathrm{Mecca} \mathrm{C}$ et al.

F12 The Association between Gastrin and Glucose Serum Concentration in Hypergastrinemic Patients with Gastric Neuroendocrine Tumors Type 1 and ECL-Cells Hyperplasia Moschouris P et al.

F13 Circulating Neuroendocrine Gene Transcripts (NETest) Decrease Early after Radical Surgery: Preliminary Results of a Prospective Study

Partelli S et al.

F14 Assessment of the Serum Levels of the Soluble Vascular Cellular Adhesion Molecule-1 (sVCAM-1) in Patients with Neuroendocrine Neoplasms

Rosiek $\mathrm{V}$ et al.
F15 Alpha-Fetoprotein in Gastro-Entero-Pancreatic Neuroendocrine Neoplasms: A Single Centre Study

Rossi RE et al.

F16 Chromogranin A as a Marker in the Follow-up of Gastro-EnteroPancreatic Neuroendocrine Neoplasms (GEP-NENs). A Systematic Review

Rossi RE et al.

F17 Development of Multiplex Biomarker Assay to Subtype Pancreatic Neuroendocrine Tumors (PanNETs) with Distinct Prognosis and Mutations Sadanandam A et al.

F18 24 Hour Urinary 5-Hydroxyindoleacetic Acid Levels in Patients with Non-Functioning Pancreatic Neuroendocrine Tumors

Tirosh $\mathrm{A}$ et al.

F19 Prognostic Value of Immunohistochemical Expression of Somatostatin Receptors (SS-R2 and SS-R5) in the Gastroenteropancreatic Neuroendocrine Tumors, Diagnosed in the University Hospital of Pleven, Bulgaria, from the Period 2010 to November 2017

Vladova $\mathrm{P}$ et al.

\section{G Imaging and Interventions (Radiology, Endoscopy)}

G01 Comparison of Glucagon-Like-Peptide-1 (GLP-1) Receptor PET/ CT, SPECT/CT and 3T MRI for the Localisation of Insulinomas: Evaluation of Accuracy in a Prospective Crossover Imaging Study Antwi A et al.

G02 Progression of Mesenteric Metastasis in Small Intestinal Neuroendocrine Tumors

Blazevic A et al.

G03 Prognostic Impact of CHOI Response Criteria Compared to RECIST in Grade 1-2 Advanced Pancreatic Neuroendocrine Tumors (CRIPNET-GETNE Study-NCT02841865)

Carmona-Bayonas A et al.

G04 99mTc-Colloid Scintigraphy in the Differential Diagnosis of Pancreatic NET

Chiloiro $\mathrm{S}$ et al.

G05 Pre-Treatment Tumor Growth Rate (TGR0) in Patients Diagnosed with Well-Differentiated Neuroendocrine Tumors (NETs) Treated with Systemic Therapies: Subgroup Analysis of the GREPONET Study

Crona J et al.

G06 How to Perform Contrast Enhanced Oncologic Staging MRI of Abdomen and Pelvis with a Hepatocyte Specific Agent in a Large Bore MR Scanner: Optimizing Extrahepatic Tumor Evaluation in Neuroendocrine Neoplasms

Fehrenbach U et al.

G07 Transcatheter Arterial Chemoembolization Synergizes with Simultaneous Microwave Ablation in the Treatment of Unresectable Gastroenteropancreatic Neuroendocrine Tumors with Liver Metastasis

Gao S et al.

G08 Linked Colour Imaging Increases the Diagnostic Yield of Type 1 Gastric Carcinoid

Gulati $S$ et al. 
maging Modality Depending on the Levels of Tumor Markers Iorio J et al.

G10 4D Ultrasound High Definition Flow with Spatio-Temporal Image Correlation in Evaluation of Neuroendocrine Liver Metastases Preliminary Study

Janas K et al.

G11 CT-Guided HDR Brachyterapy in Patients with Advanced NonResectable and Progressive NEN with Liver Bulky Disease. Pilot Study

Kieszko D et al.

G12 Value of Tumor Growth Rate (TGR) as an Early Predictor of Patients' Outcome in Patients Diagnosed with WellDifferentiated Neuroendocrine Tumors (NETs): The GREPONET Study

Lamarca A et al.

G13 Hepatic Tumor Burden Can Be Reduced Rapidly and Effectively by Trans-Arterial Embolization Combined with Systemic Therapy in Patients with Neuroendocrine Tumor Liver Metastasis (NETLM)

Liu Y et al.

G14 Carcinoid Heart Disease - Diagnostic Usefulness of Cardiac Computed Tomography - A Pilot Study Michałowska I et al.

G15 Mechanisms of Main Pancreatic Duct Dilatation in Pancreatic Neuroendocrine Tumors (PanNETs): Radiopathologic Correlation Ronot $\mathrm{M}$ et al.

G16 The RECIST Criteria Compared to Conventional Response Evaluation after Peptide Receptor Radionuclide Therapy in Patients with Neuroendocrine Neoplasms

Thiis-Evensen E et al.

G17 The Role of Magnetic Resonance Imaging and Endoscopic Ultrasound Imaging in the Diagnosis and Management of Pancreatic Neuroendocrine Tumors (pNET) in Patients with Multiple Endocrine Neoplasia Type 1 (MEN1)

Tsoli $\mathrm{M}$ et al.

\section{H Medical Treatment - Chemotherapy, Somatostatin Analogues, Interferon}

H01 Intravenous versus Oral Etoposide: Efficacy and Correlation to Clinical Outcome in Patients with High-Grade Gastroenteropancreatic Neuroendocrine Neoplasms (WHO G3) Ali AA et al.

H02 Phase 1 Pharmacokinetic and Pharmacodynamic Study of APOC, a New Controlled Release Formulation (CRF) of $15 \mathrm{mg}$ Octreotide Acetate in Healthy Male Volunteers

Antonijoan Arbós RM et al.

H03 Prospective, Multi-Center, Open Label, Phase Study II of APOC, a New Controlled Release 15mg Octreotide Acetate, for the Treatment of Advanced NETs

Capdevila J et al.

H04 Clinical Use of Lanreotide AG as Initial Therapy in Patients with Advanced, Non-Resectable Well (G1) and Moderate (G2) Differentiated Pancreatic Neuroendocrine Neoplasms (p-NEN) Ćwikła J et al.
H05 Sequential Therapies in Well-Differentiated Gastrointestinal Neuroendocrine Tumors G3 (NET G3): Platinum-Based vs. Platinum-Free Regimens Dal Buono A et al.

H06 Ex Vivo Activity of Cytotoxic Drugs and Targeted Agents in Small Intestinal Neuroendocrine Tumors

Daskalakis K et al.

H07 Audit of a New Nurse-Led Non-Medical Prescribing (NMP) Clinic for Systemic Anti-Cancer Agents (SACTs)

Davies $\mathrm{P}$ et al.

H08 Efficacy and Toxicity of Metronomic Temozolomide in SecondLine Treatment of Advanced Neuroendocrine Neoplasms De Divitiis C et al.

H09 Therapeutic Sequences in Patients with G1-G2 Neuroendocrine Tumors (NETs): An Observational, Multicentre, Prospective/ Retrospective Study Faggiano A et al.

H10 Interim Analyses LANREL07484: Quality of Life in Patients with Symptomatic Gastroenteropancreatic Neuroendocrine Tumors Treated with Lanreotide Autogel in South Africa Geldenhuys D et al.

H11 Interim Analyses LANREL07484: Response to Treatment and Self-Reported Satisfaction in Symptoms in Patients with Gastroenteropancreatic Neuroendocrine Tumors Treated with Lanreotide Autogel in South Africa Geldenhuys D et al.

H12 Therapeutic Efficacy of Platinum/Etoposide Regimens in the Treatment of Advanced Poor Differentiated Neuroendocrine Carcinomas of the Lung: A Retrospective Analysis Giuffrida D et al.

H13 Phase II Study of Temozolomide Monotherapy in Patients of Neuroendocrine Carcinoma with Resistant to Platinum-Based Chemotherapy Ichikawa Y et al.

H14 A Comparison of Retrospective Database Analysis with Chart Review in Patients Receiving Somatostatin Analog (SSA) in Neuroendocrine Tumors (NETs)

Klink A et al.

H15 Efficacy of Octreotide LAR in Treatment of Naive Patients with Advanced, Non-Resectable Well and Moderate Differentiated Pancreatic Neuroendocrine Neoplasms (p-NENs) Kolasińska-Ćwikła A et al.

H16 International Survey of Clinical Practice Exploring Use of Platinum-Etoposide Chemotherapy for Extra-Pulmonary High Grade Neuroendocrine Carcinoma (EP-G3-NEC)

Lamarca A et al.

H17 Antiproliferative Effect of Above-Label Doses of Somatostatin Analogues (SSA) for the Management of Neuroendocrine Tumors (NETs)

Laskaratos $\mathrm{F}$ et al.

H18 Predictors of Antiproliferative Effect of Lanreotide Autogel (LA) as First-Line Therapy for Advanced Neuroendocrine Tumors (NETs)

Laskaratos $\mathrm{F}$ et al. 
H19 Disease Control in Progressive Pancreatic and Intestinal Neuroendocrine Tumors by Combined Treatment with Lanreotide Autogel and Temozolomide: The SONNET Study Pavel $\mathrm{M}$ et al.

H20 Perioperative Chemotherapy in Resectable Neuroendocrine Carcinomas of the Digestive Tract

Pellat A et al.

H21 Role of Interval Reduction of Somatostatin Analogs in Patients with Progressive Neuroendocrine Tumors: Our Experience Puliafito I et al.

H22 Evaluation of Streptozocin-Based Chemotherapeutic Regimens for Advanced Pancreatic Neuroendocrine Tumors: A Multi-Center Clinical Study in Japan

Sakamoto Y et al.

H23 Efficacy of Oral Chemotherapy with Capecitabine and Temozolomide (Captem) in Patients with Metastatic Neuroendocrine Tumors (NETS). A Single-Institution Experience Smiroldo V et al.

H24 Combination of Capecitabine and Temozolomide for Advanced Thymic Neuroendocrine Tumors Wang X et al.

H25 Capecitabine/Temozolomide (CAPTEM) Regimen in the Treatment of Advanced Neuroendocrine Neoplasms: A SingleCenter Retrospective Study in China

Zhang $\mathrm{Y}$ et al.

\section{Medical Treatment - Targeted Therapies}

101 A Pilot Study of the Cyclin Dependent Kinases 4, 6 Inhibitor Ribociclib in Patients with Foregut Neuroendocrine Tumors Dasari A et al.

102 Do We Need Hormonal Therapy for Pancreatic Neuroendocrine Tumors? An Effort to Reduce the Size of PanNETs Fatima A et al.

103 Relationship between Metabolic Toxicity and Efficacy of Everolimus in Patients with Neuroendocrine Tumors (NETs): A Pooled Analysis from the Randomized, Phase 3 RADIANT-3 and RADIANT-4 Trials Fazio $\mathrm{N}$ et al.

104 Activity of Bevacizumab in Neuroendocrine Neoplasms Fejzibegovic $\mathrm{N}$ et al.

105 Phase II Trial of Cabozantinib in Patients with Carcinoid and Pancreatic Neuroendocrine Tumors Chan J et al.

106 Efficacy of Sunitinib Correlated with Clinical, Radiological Variables, Dose-Intensity and Treatment Time in Advanced Grade 1-2 Pancreatic Neuroendocrine Tumors (CRIPNET-GETNE Study NCT02841865)

Jimenez-Fonseca P et al.

107 Evaluation of Everolimus (EVE) in Patients with Metastatic Lung Neuroendocrine Tumors

Kuznetsova A et al.
108 OPALINE Study: Observational Study in a Real-World Setting of the Systemic Treatment of Progressive Unresectable or WellDifferentiated Metastatic Pancreatic Neuroendocrine Tumors (pNET)

Lombard-Bohas C et al.

109 Sunitinib in Patients with Pre-Treated Pancreatic Neuroendocrine Tumors: A Real-World Study Rinzivillo $\mathrm{M}$ et al.

I10 ElevatION: NET-201 A Phase II Study to Evaluate the Efficacy and Safety of PDR001 in Patients with Metastatic, Well-Differentiated NET of Pancreatic/GI/Thoracic Origin or Poorly-Differentiated GEP NEC Who Have Progressed on Prior Treatment Yao JC et al.

\section{J Medical Treatment - Others, Not Specified}

J01 Outcomes of Locoregional Treatment for Unifocal Progression in Widespread Metastatic Gastroenteropancreatic Neuroendocrine Tumors

Al-Toubah T et al.

J02 Clinical Characteristics and Prognostic Analysis of 14 Patients with Gastric Mixed Adenoneuroendocrine Carcinoma Bei X et al.

J03 Metastatic Neuroendocrine Neoplasia Treatments in over 70 Years Old Patients: A Retrospective Outcome Analysis Bongiovanni A et al.

J04 The Role of Diagnostic Biopsy to Determine the Treatment Tactics of Patients with Lung Tumors of Different Histogenesis. The Experience of a Single Multidisciplinary Center for the Period 2014-2017 Years

Byakhova $\mathrm{M}$ et al.

J05 On-going Evaluation of the Use of Resources and the Costs (UR/C) Associated with Controlled or Uncontrolled Carcinoid Syndrome (CS) in Patients (pts) with Neuroendocrine Tumors (NETs): RECOSY Study Design

Custodio A et al.

J06 Time to Sustained Improvement in Bowel Movement Frequency with Telotristat Ethyl: Analyses of Two Phase 3 Studies in Carcinoid Syndrome Dillon J et al.

J07 Identifying the Severity of Psychosocial Symptoms among Patients Diagnosed with Pancreatic Neuroendocrine Tumor. Do We Really Need Emotional Support Groups?

Fatima A et al.

J08 Clinical Utility (CU) Evaluation of the Health-Related Quality-of-Life (HRQoL) QLQ-GINET21 Questionnaire (QNR) in the Treatment of Patients (pts) with Gastrointestinal (GI) Neuroendocrine Tumors (NETs). QUALINETS Study Gallego J et al.

J09 OPERA: Observational Study of Perception of Information and Quality of Life in Patients with Neuroendocrine Tumors Starting Lanreotide - Study Design

Gueguen D et al. 
Durvalumab plus Tremelimumab for the Treatment of Patients (pts) with Advanced Neuroendocrine Neoplasms (NENs) of Lung or Gastroenteropancreatic (GEP) Origin. A Phase II Multicohort Trial (DUNE Trial / GETNE 1601)

Hernando-Cubero J et al.

J11 Clinical and Morphological Features of Extrapulmonary SmallCell Cancer

Ivanov A et al.

J12 Efficacy and Safety of Anti-PD-1 Antibody (IBI308) in Treating Advanced Neuroendocrine Neoplams

Jia R et al.

J13 Patient and Clinician Perspectives on Symptom Priorities Across the Spectrum of Neuroendocrine Tumors (NETs)

Kaiser $\mathrm{K}$ et al.

J14 Exploring Nutrition Screening and Management Practices amongst Health Professionals Managing Patients with Neuroendocrine Tumors

Kennedy E et al.

J15 Nutritional Status and Considerations for Patients Diagnosed with a Gastroenteropancreatic Neuroendocrine Tumor: Preliminary Baseline Characteristics from the Nutrition in NETs Study

Kennedy E et al.

J16 Development of a Patient-Centred Service for Neuroendocrine Tumors (NETs) in Wales: Population Based National

Commissioning

Khan MS et al.

J17 Oral Ondansetron Offers Effective Symptomatic Bridging for Carcinoid Syndrome Refractory to Somatostatin Analogues Kiesewetter B et al.

J18 Systemic Anti-Cancer Therapies in Neuroendocrine Tumor Patients Impair Nutritional Status

Pevny $\mathrm{S}$ et al.

J19 Menstrual Dysfunction in Women with Prolactinomas Safarova M et al.

J20 Safety and Efficacy of TAE and SIRT in NET Patients Van Veenendaal L et al.

\section{K Nuclear Medicine - Imaging and Therapy (PRRT)}

K01 Influence of Lanreotide on Uptake of [68Ga]-DOTATATE in Patients with NETs

Aalbersberg E et al.

K02 Parameters to Predict Overall Survival after PRRT A Multivariate Analysis in 783 Patients

Aalbersberg E et al.

K03 Predicting Response to PRRT: Development and Validation of a Blood-Based Predictive Biomarker

Bodei L et al.

K04 International Multicentre Retrospective Study on the Safety of Radioembolization with Yttrium-90 Resin Microspheres after Systemic Radionuclide Therapy in Neuroendocrine Tumors Braat A et al.
K05 Detection of Bone Metastases at FDOPA-PET in Small-Intestine (si)NET: Prevalence and Associated Factors de Mestier L et al.

K06 $213 \mathrm{Bi}$ and Ac 225 DOTATOC Receptor Labeled Targeted AlphaRadionuclide Therapy in Neuroendocrine Tumors Refractory to Beta Radiation - Early Experience

Dureja S et al.

K07 Short-Term Change in Symptoms and Adverse Events Evaluation after PRRT - First Experience after 56 Patients Huizing $\mathrm{D}$ et al.

K08 PRRT in Hindgut and Cancer of Unknown Primary NEN Kolasińska-Ćwikła A et al.

K09 Favourable Outcomes of Peptide Receptor Radionuclide Therapy (PRRT) for Treatment of Metastatic Rectal Neuroendocrine Neoplasia (NEN)

Kong $\mathrm{G}$ et al.

K10 Value of Somatostatin Receptor Scintigraphy (SRS) in Patients with Appendiceal Neuroendocrine Neoplasms (ANEN) Based on Clinical Follow-up and Results of NETest

Konsek S et al.

K11 Positron Emission Tomography (PET) Predictors of Tumor Response to Peptide Receptor Radionuclide Therapy (PRRT) in Metastatic Neuroendocrine Tumors (NET)

Ladwa $\mathrm{R}$ et al.

K12 Pretherapeutic Predictors of Tumor Absorbed Dosimetry in Radionuclide Therapy for Metastatic Neuroendocrine Tumors Ladwa R et al.

K13 Tumor Absorbed Dosimetry and Response of Radionuclide Therapy in Metastatic Neuroendocrine Tumor Ladwa $\mathrm{R}$ et al.

K14 Diagnostic Utility of Ki-67 as a Mean to Predict the Uptake of Functional Imaging Modalities in Patients with Neuroendocrine Neoplasms

Liotsou T et al.

K15 Peptide Receptor Radionuclide Therapy (PRRT) in 35 Patients with Metastatic Neuroendocrine Neoplasms (NENs): Overall Response and Toxicity Liotsou $\mathrm{T}$ et al.

K16 Education and Preparation for Nuclear Medicine Procedures in Neuroendocrine Tumor Patients Mailman J et al.

K17 Nonfunctional Pancreatic Neuroendocrine Tumor (NF PNET) Imaging and Evaluation Using 18F-FDG and 68Ga-DOTANOCPET/CT: Initial Data of a Prospective Study Majala $S$ et al.

K18 One Year Experience with Lutetium-DOTATATE for Disseminated NETs in the Gregorio Marañon Hospital Picallo $\mathrm{M}$ et al.

K19 Imaging of Advanced Medullary Thyroid Carcinoma with the CCK-2 Receptor Agonist 177Lu-PP-F11N - Preliminary Proof of the Principle within the "Lumed" Study

Rottenburger $\mathrm{C}$ et al. 
K20 Peptide Receptor Radionuclide Therapy (PRRT) in Gastroenteropancreatic Grade 3 Neuroendocrine Neoplasms: A Retrospective International Multicenter Study Skovgaard D et al.

K21 Does Somatostatin Receptor (SSR) Positive Tumor Volume Determined on Ga68 DOTANOC PET/CT in Patients with Paraganglioma (PGL)/Pheochromocytoma(PCC) Correlate with Biomarkers? An Explorative Study

Steyn R et al.

K22 Overall Survival, Progression-Free Survival, and Quality of Life Updates from the NETTER-1 Study: 177Lu-Dotatate vs. High Dose Octreotide in Progressive Midgut Neuroendocrine Tumors Strosberg J et al.

K23 A Randomized Controlled Study Comparing Treatment of Gastro-Entero-Pancreatic Neuroendocrine Tumors (GEPNET) with 177Lu-DOTATATE Alone and in Combination with Capecitabine

Van der Zwan W et al.

K24 PFS and OS after Salvage Peptide Receptor Radionuclide Therapy (PRRT) with 177Lu-DOTATATE in Patients with Gastroenteropancreatic or Bronchial Neuroendocrine Tumors (GEP-NETs) - The Rotterdam Cohort

Van der Zwan W et al.

K25 Study to Evaluate the Optimal Dose of 68Ga-OPS202 as a PET Imaging Agent in Patients with GEP-NETs

Virgolini I et al.

K26 Optimizing Reconstruction Algorithm to Improve Quality of Post-PRRT Yittrium-90 PET Scan

Yan SX et al.

K27 Efficacy of Adding Somatostatin Analogues to [177Lu]LuOctreotate as a Combination and Maintenance Therapy in Metastatic Neuroendocrine Tumors Yordanova A et al.

K28 The Correlation between 68Ga-DOTATATE PET/CT Results and Tumor Proliferation in Patients with Gastroenteropancreatic Neuroendocrine Neoplasms (GEP-NENs)

Yu J et al.

\section{Surgical Treatment and Ablative Therapies}

L01 Palliative Surgery in Advanced Small Intestinal Neuroendocrine Tumors

Blazevic A et al.

L02 Morphological Factors Related to Nodal Metastases in Neuroendocrine Neoplasms of the Appendix. A Multicenter Retrospective Study

Brighi $\mathrm{N}$ et al.

L03 Appropriate Surgical Strategy in Appendiceal Neuroendocrine Tumors: Is Right Hemicolectomy Oncologically Justified or Overtreatment?

Clift AK et al.

L04 The Prognostic Impact of Primary Tumor Resection in Pancreatic Neuroendocrine Tumors with Synchronous Multifocal Liver Metastases

Dai H et al.
L05 Neuroendocrine Neoplasms of the Appendix: Characterization of 251 Patients Referred to the Copenhagen NET Centre of Excellence

Holmager $\mathrm{P}$ et al.

L06 Prognostic Value of Lymph Node Status and Extent of Lymphadenectomy in Non Functioning Pancreatic Neuroendocrine Tumors: Outcome Analysis from 378 Consecutive Resections in a High-Volume Institution Malpaga A et al.

L07 A Matched-Pair Analysis of Conventional Surgical Methods versus Enucleation for Pancreatic Neuroendocrine Tumors Mao W et al.

L08 The Evolution of Surgical Strategies for Pancreatic Neuroendocrine Tumors (Pan-NENs): Time-Trend and Outcome Analysis from 587 Consecutive Resections at a High-Volume Institution Marchegiani G et al.

L09 A Comparison of Recurrence after Curative Resection between Pancreatic and Duodenal Neuroendocrine Tumors Masui $\mathrm{T}$ et al.

L10 35 Years of Experience in a Single Center on Distal Pancreatectomy for Neuroendocrine Tumors Milanetto AC et al.

L11 Health-Related Quality of Life Determinants in Swedish Patients after Surgery for Small Intestinal Neuroendocrine Tumors Milanetto AC et al.

L12 Pancreatic Involvement in Small Intestinal Neuroendocrine Tumors

Milanetto AC et al.

L13 Pancreatic NET or Pancreatic Clear Cell Renal Cancer Metastases in Post-Nephrectomy Patients?

Milanetto AC et al.

L14 Pancreatic NETs with Liver Metastases. Outcome of Surgically Treated Patients. A Single Center Experience Milanetto AC et al.

L15 Survival after Surgical Treatment of Small Intestinal Neuroendocrine Tumors with Liver or Peritoneal Metastases: 25 Years of Experience Milanetto AC et al.

L16 Tumor Size Correlates with Grading in Nonfunctioning Pancreatic Neuroendocrine Tumors and Is Not Age-Dependent Muffatti F et al.

L17 Predicting Resectability of Primary Tumor and Mesenteric Lumps in Patients with Small Intestine Neuroendocrine Tumors Piccioli AN et al.

L18 Liver Resection for Neuroendocrine Tumors Liver Metastases in Transplantable Patients within the Milan Criteria Ruzzenente A et al.

L19 The Laparoscopic Approach in the Surgical Treatment of the Gastric Neuroendocrine Tumors Zubaryev $\mathrm{M}$ et al. 


\section{Non Digestive NETs (Bronchial, Thymic, Others) - Diagnosis and Therapy}

M01 Clinical Characteristics, Treatment Outcomes and Potential Novel Therapeutic Options for Patients with Prostatic Neuroendocrine Carcinoma Apostolidis L et al.

M02 Pulmonary Function Test Physiology and Progression in Diffuse Idiopathic Pulmonary Neuroendocrine Cell Hyperplasia Barlow J et al.

M03 Primary Neuroendocrine Tumors of the Breast Castillo-Fernandez $\mathrm{O}$ et al.

M04 Results of 252 Patients with Bronchopulmonary Neuroendocrine Tumors Treated at the Copenhagen NET Centre of Excellence Groendahl V et al.

M05 PD-L1 Expression and Its Clinical Relevance in Neuroendocrine Tumors of the Lung

Kasajima A et al.

M06 Clinicopathologic Features and Treatment Outcome of 225 Newly Diagnosed Pulmonary Carcinoids: A Single Center Experience of 28 Years

Li Q et al.

M07 Large Cell Neuroendocrine Carcinoma of the Lung: Single-Centre Retrospective Cohort Study

Martins Branco D et al.

M08 Typical and Atypical Bronchial NETs with Advanced Disease: Incidence, Management and Survival

McFadyen R et al.

M09 Validation of a Blood-Based Biomarker Test for the Diagnosis and Management of Bronchopulmonary Neuroendocrine Tumors

Modlin I et al.

M10 A Comparison of Diagnostic and Management Pathways for Patients with Lung Neuroendocrine Tumors in ENETS Centres of Excellence vs Non-Accredited Centres in the UK: Results from the National Lung NET Pathway Project ('LEAP')

Talbot D et al.

\section{N Endocrine Malignancies (MTC, Pheochromocytoma) - Diagnosis and Therapy}

N01 Clinical and Pathological Differences between NEC and Carcinoma of Esophagus: A Population Based Study Cai $\mathrm{W}$ et al.

N02 Sensitivity and Specificity of Insulin, C-Peptide and Nadir Glucose during $72 \mathrm{hr}$ Supervised Fast in Diagnosis of Insulinoma Loh W J et al.

N03 Preliminary Safety and Efficacy of Rovalpituzumab Tesirine in Patients with Delta-Like Protein 3-Expressing Advanced Solid Tumors

Mansfield A et al.

N04 Early Prognostic Factors in Medullary Thyroid Carcinoma Muñoz de Nova JL et al.

N05 Pancreatic Neuroendocrine Tumor in Polish Population with MEN 1 Syndrome

Soczomski $\mathrm{P}$ et al.

N06 Clinical Analysis of 15 Cases of Gallbladder Neuroendocrine Carcinoma

Zhang Y et al. 